\title{
Abnormal Anatomical and Radiological Changes in a Rare Presentation of Adult Developmental Dysplasia of the Hip
}

\author{
Jnana Aditya Challa ${ }^{1}$, Rafid Kasir ${ }^{2}$, Varna Taranikanti ${ }^{1}$ \\ 1. Foundational Medical Studies, Oakland University William Beaumont School of Medicine, Rochester, USA 2. \\ Department of Orthopedic Surgery, Beaumont Health System, Royal Oak, USA
}

Corresponding author: Varna Taranikanti, taranikanti@oakland.edu

\begin{abstract}
Adult presentation of bilateral dysplasia and dislocation is an extremely rare presentation. The management of adult hip dysplasia is to preserve the hip and reduce pain through surgical intervention. Hence, early diagnosis provides more options as the treatment dilemma with the late presentation is very complicated with debatable prognosis. The case presented is a 53 -year old woman who complained of persistent pain in the hip region. On radiology, dysplasia and dislocation of both the hip joints were observed along with soft tissue abnormalities around the joint. In this case report, we discuss the underlying pathophysiology that might have led to the abnormal radiological and anatomical changes in the hip region and the possible treatment options in a conservatively managed case of developmental dysplasia of the hip (DDH).
\end{abstract}

Categories: Orthopedics, Anatomy

Keywords: developmental dysplasia, hip dislocation, radiological changes, treatment dilemma

\section{Introduction}

Hip dysplasia is a developmental defect of the hip joint where there is anomalous development of the osseous and soft tissue structures around the hip joint [1]. Developmental dysplasia of the hip (DDH) is the most common orthopedic disorder in newborns $(1: 100)$ and is more common in first-born females $(5 \mathrm{~F}: 1 \mathrm{M})$. However, complete dislocation is more commonly seen in adults as compared to children; thus early diagnosis of DDH is extremely important for therapeutic success. There have been very few reports of adult $\mathrm{DDH}$ as a consequence of irregular long-term follow-up of childhood DDH [2]. The treatment dilemma with the late presentation is very complicated with a debatable prognosis. Herein we report a 53-year-old woman who presented with persistent pain in the right groin and gluteal region radiating to the distal thigh. Radiology confirmed developmental dysplasia of the hip joint with complete dislocation. In this case report, we discuss the underlying pathophysiology that might have led to the abnormal radiological and anatomical changes in the hip region and the possible treatment options in a conservatively managed case of DDH.

Received 07/14/2020

Review began $07 / 27 / 2020$ Review ended 08/08/2020 Published 08/22/2020

\section{() Copyright 2020}

Challa et al. This is an open access article distributed under the terms of the Creative Commons Attribution License CC-BY 4.0., which permits unrestricted use, distribution, and reproduction in any medium, provided the original author and source are credited.

\section{Case Presentation}

A 53-year-old woman came to the Beaumont Orthopaedic Institute (BOI) Joints clinic with persistent pain in the right groin and gluteal region radiating to the distal thigh for over a year. The symptoms were not preventing her from doing routine activities. She denied numbness or tingling and has been undergoing physical therapy for this pain for a year, which she reports did not help. Her previous surgical history revealed valgus producing osteotomy with bilateral femoral plates and subsequent removal of the plate on the right side. Physical examination revealed a conscious and mentally alert woman who was able to ambulate without a significant limp. The hip examination findings are shown in Table 1.

\begin{tabular}{|l|l|l|}
\hline \multicolumn{2}{|c|}{ Right } & Left \\
\hline Movements & Hip flexion to $80^{\circ}$; internal rotation $30^{\circ}$; external rotation $30^{\circ}$ & Hip flexion to $80^{\circ}$; internal rotation $45^{\circ}$; external rotation $45^{\circ}$ \\
\hline Tone & Knee and ankle normal \\
Sensations & Sensations Intact to light touch bilaterally \\
Pulses & Peripheral pulses palpable bilaterally \\
\hline
\end{tabular}

TABLE 1: Hip examination findings 


\section{Cureus}

Reviewed X-rays showed bilateral acetabular hip dysplasia with significant superior dislocation of bilateral femoral heads and advanced degenerative changes. Figure 1 shows anomalous development of the osseous and soft tissue structures around the hips bilaterally. The osseous component involved in the right acetabulum is underdeveloped and defective: it is angular in shape, smaller in size, and decreased in depth.

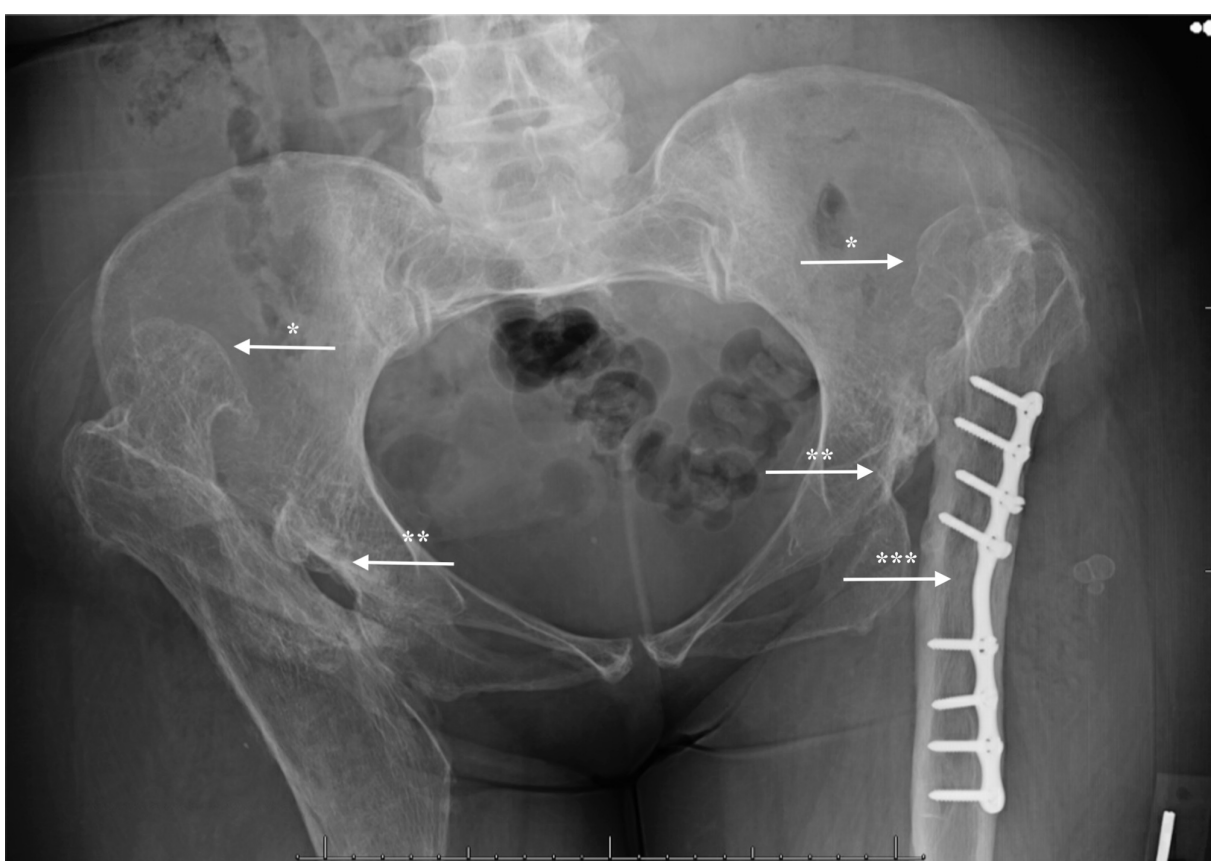

\section{FIGURE 1: Anteroposterior view showing bilateral femoral dysplasia}

The femoral head and neck are dysplastic (*) with coxa valga deformity (increased caput collum diaphysea angle/femoral neck anteversion angle), shallow acetabular socket $\left(^{* \star}\right)$ and femoral plate $\left({ }^{* * \star}\right)$.

X-rays: courtesy of Beaumont Royal Oak Hospital.

\section{Discussion}

DDH is a complex disorder mainly caused by the incongruity between the head of the femur and the abnormal socket of the acetabulum. The disease spectrum ranges from mild dysplasia to dislocation of the hip secondary to capsular laxity and mechanical factors (such as movements occurring from hip flexion to extension $[3,4]$.

In this case report, we described a post-surgical patient who has previously been treated with valgus producing osteotomy with bilateral femoral plates for hip deformity. The femoral plate on the right side has since been removed and based on history, physical examination and radiology her condition has been diagnosed as adult dysplasia of the hip.

During embryonic development, limb buds begin to form in the fourth week. In the eighth week of gestation, the hip joint starts developing with the appearance of the acetabulum and femoral head. Soft tissue structures around the hip joint occur during the 12th week and muscles and ligaments start to develop at around 18th weeks. DDH commonly occurs during the last four weeks of fetal growth and perinatally because of mechanical forces acting on the fetus [5].

In our patient, the osseous component involved was the acetabulum, which was underdeveloped and defective. This can primarily be attributed to a defect in the proximal femoral epiphyseal plate as it plays an important role in the development of the hip joint. Gage et al. studied the effects of trochanteric epiphyseodesis on the growth of the proximal end of the femur following necrosis of the capital femoral epiphysis and concluded that insult to the epiphysis in infancy results in a mean growth loss in the proximal end of the femur of 21.5 millimeters with trochanteric epiphyseodesis [6].

Furthermore, as the femur is displaced superiorly in this case, the shear forces of muscles (traction epiphysis) generate pulling forces in angles different from normal. This changes the shape and growth of proximal femur and acetabulum, leading to the hip subluxation/dislocation. Furthermore, a pseudo acetabulum has most likely formed in order to stabilize the displaced femur and enable articulation. This pseudo acetabulum is made up of scar tissue and is further stabilized by muscles acting on the joint, the shadow of which is visible in Figure 1 outlined by the femoral head. As Odak et al. discuss in their paper, the 
presence of a well-defined, concentric, radio-opaque shadow around the dislocated femoral head is suggestive of a pseudo acetabulum [7]. Although this pseudo acetabulum in their case was formed following a total hip arthroplasty in an elderly patient with a history of recurrent dislocations, the characteristics of the radiological findings suggest a similar prognosis in our patient. Moreover, inappropriate posture and predominant weight bearing on the right side could also cause greater compression of the femoral shaft on the ipsilateral side, thinning of the compact bone, and widening of the medullary cavity. Ultimately these changes, combined with the plates that were removed from the right side, resulted in a widened and inappropriately angled femoral shaft that is weaker than normal as seen in Figure 1.

Lastly, as our patient is a 53-year-old post-menopausal woman, hormonal changes may have resulted in osteomalacia in the femoral shaft leading to the recent onset of pain in the hip region. According to the study by Ji et al., the mean age of natural menopause is 51 years and results in decreased production of estrogen, which impairs the normal bone turnover cycle [8].

As a result of the aforementioned late presentation of DDH in our patient, the treatment is very complicated with debatable prognosis. Ideally, early diagnosis of DDH (at or before six months of age) is extremely important for therapeutic success as Pavlik bracing would be a viable option. Pavlik bracing is a soft dynamic harness that could be used to treat DDH in infants up to six months old. It maintains hip in flexion and abduction [9]. Due to the age and high degree of dysplasia (Crowe type IV) in our patient, the only viable surgical intervention would be total hip replacement [10]. This would involve subtrochanteric shortening osteotomy: excision of femur $4 \mathrm{~cm}$ below the head bilaterally and attachment of head to the shaft. Also, the acetabular socket will have to be widened to enable articulation. Finally, a total hip replacement will be performed bilaterally with an appropriately sized socket $(\sim 38 \mathrm{~mm})$ and femoral head $(\sim 22 \mathrm{~mm})$.

\section{Conclusions}

DDH in adults is extremely uncommon. Early diagnosis of DDH is extremely important for therapeutic success. The treatment dilemma with late presentation is very complicated with debatable prognosis. As our patient was able to ambulate and is managing her activities of daily living adequately, surgical intervention may potentially lead to a reduced quality of life. As the risks of surgery may outweigh the benefits at this stage, she can be conservatively managed without surgery or any additional treatment other than continuing physical therapy and over the counter analgesics with follow up as needed if symptoms worsen.

\section{Additional Information \\ Disclosures}

Human subjects: Consent was obtained by all participants in this study. Conflicts of interest: In compliance with the ICMJE uniform disclosure form, all authors declare the following: Payment/services info: All authors have declared that no financial support was received from any organization for the submitted work. Financial relationships: All authors have declared that they have no financial relationships at present or within the previous three years with any organizations that might have an interest in the submitted work. Other relationships: All authors have declared that there are no other relationships or activities that could appear to have influenced the submitted work.

\section{References}

1. Zamborsky R, Kokavec M, Harsanyi S, Attia D, Danisovic L: Developmental dysplasia of hip: perspectives in genetic screening. Med Sci. 2019, 7:59. 10.3390/medsci7040059

2. Loder RT, Skopelja EN: The epidemiology and demographics of hip dysplasia . ISRN Orthop. 2011, 2011:238607. 10.5402/2011/238607

3. Developmental dysplasia of the hip (DDH) . (2020). Accessed: 2020: https://www.orthobullets.com/pediatrics/4118/developmental-dysplasia-of-the-hip-ddh .

4. Murray CJL, Atkinson C, Bhalla K, et al.: The state of US Health, 1990-2010. JAMA. 2013, 310:591-606. 10.1001/jama.2013.13805

5. Lee MC, Eberson CP: Growth and development of the child's hip. Orthop Clin North Am. 2006, 37:119-132. 10.1016/j.ocl.2005.12.001

6. Gage JR, Cary JM: The effects of trochanteric epiphyseodesis on growth of the proximal end of the femur following necrosis of the capital femoral epiphysis. J Bone Joint Surg Am. 1980, 62:785-794.

7. Odak S, Mangwani J, Ivory J: Pseudoacetabulum a subtle radiological feature of a chronic dislocated total hip replacement. BMJ Case Rep. 2012, 2012: 10.1136/bcr-2012-006686

8. Ji M-X, Yu Q: Primary osteoporosis in postmenopausal women. Chronic Dis Transl Med. 2015, 1:9-13. 10.1016/j.cdtm.2015.02.006

9. Pavlik Harness: AliMed ${ }^{\circledR}$ Pavlik-Type Harness. Accessed: 30/06/2019: https://www.alimed.com/alimedpavlik-harness.html.

10. Chen M, Gittings DJ, Yang S, Liu X: Total hip arthroplasty for Crowe type iv developmental dysplasia of the hip using a titanium mesh cup and subtrochanteric femoral osteotomy. Iowa Orthop J. 2018, 38:191-195. 